\title{
Perioperative use of crystalloids in patients undergoing open radical cystectomy: balanced Ringer's maleate versus a glucose 5\%/potassium-based balanced solution: study protocol for a randomized controlled trial
}

Lukas M Löffel', Bettina Kleeb ${ }^{1}$, Fiona C Burkhard² and Patrick Y Wuethrich ${ }^{1 *}$

\begin{abstract}
Background: The optimal crystalloid solution to use perioperatively in patients undergoing open radical cystectomy remains unclear. Many of the fluids used for intravenous hydration contain supraphysiologic concentrations of chloride, which can induce hyperchloremia and metabolic acidosis, resulting in renal vasoconstriction and decreased renal function. In addition, patients receiving less fluid and less sodium show faster recovery of gastrointestinal (Gl) function after colonic surgery.

Methods and design: This is an investigator-initiated, single-center, randomized, controlled, parallel group trial with assessor-blinded outcome assessment, in the Department of Urology, University Hospital Bern, Switzerland. The study will involve 44 patients with bladder cancer scheduled for radical cystectomy and urinary diversion. The primary outcome is the duration between the end of surgery and the return of the Gl function (first defecation). Secondary outcomes are fluid balance (body weight difference postoperatively versus preoperatively) and the incidence of kidney function disorders according to the Risk - Injury - Failure - Loss - End Stage Renal Disease (RIFLE classification). An equal number of patients are allocated to receive Ringerfundin $n^{\circledast}$ solution or a glucose/ potassium-based balanced crystalloid solution as baseline infusion during the entire time that intravenous administration of fluid is necessary during the perioperative period. The randomized crystalloid solution is infused at a rate of $1 \mathrm{ml} / \mathrm{kg} / \mathrm{h}$ until the bladder has been removed, followed by $3 \mathrm{ml} / \mathrm{kg} / \mathrm{h}$ until the end of surgery. Postoperative hydration is identical in both groups and consists of $1,500 \mathrm{ml}$ of the randomized crystalloid solution per 24 hours. Postoperative patient care is identical in both groups; patients are allowed to drink clear fluids immediately after surgery, and liquid diet is started on postoperative day 1, as well as active mobilization and the use of chewing gum. Body weight is measured daily in the morning. Time of first flatus and first defecation are recorded.
\end{abstract}

Discussion: This trial assesses the benefits and harms of two different balanced crystalloid solutions for perioperative fluid management in patients undergoing open radical cystectomy with urinary diversion, with regard to return of $\mathrm{Gl}$ function and effects on postoperative renal function.

Trial registration: Current Controlled Trials ISRCTN32976792 (registered on November 21 2013).

Keywords: Crystalloid solution, Return of the gastrointestinal function, Radical cystectomy

\footnotetext{
* Correspondence: patrick.wuethrich@insel.ch

'Department of Anesthesiology and Pain Medicine, Inselspital, Bern

University Hospital, $\mathrm{CH}-3010$ Berne, Switzerland

Full list of author information is available at the end of the article
} 


\section{Background}

A recent randomized double-blind clinical trial has demonstrated that intraoperative restrictive hydration with a maleate Ringer's solution (Ringerfundin ${ }^{\circ}$ ) combined with a concomitant norepinephrine infusion reduces the incidence of postoperative complications and hospitalization time [1]. Patients to whom restrictive hydration was administered were more inclined to have a postoperative zero fluid balance (i.e. weight difference on postoperative day (POD) 1 versus preoperatively was 0 ) compared with patients in the control group, who had a positive fluid balance of approximately $2 \mathrm{~kg}$ on POD 1 . A positive postoperative fluid balance is known to be associated with increased postoperative complications [2]. In addition, the intraoperative use of norepinephrine, by minimizing the risk of fluid overload, is associated with a lower incidence of acute kidney damage [3].

Although enough evidence exists to support the rational use of perioperative restrictive hydration, the choice of the optimal crystalloid solution to use remains unclear, and intravenous chloride administration is ubiquitous [4]. Many of the fluids used for intravenous hydration contain supraphysiological concentrations of chloride, which can induce hyperchloremia and metabolic acidosis, resulting in renal vasoconstriction and decreased renal function [5]. The effect of chloride on the kidney is of concern because acute kidney injury has been associated with high mortality, and may require invasive renal replacement therapy [6,7]. After administration of $\mathrm{NaCl} 0.9 \%$, glucose $5 \%$ and Ringer's lactate solutions in normal subjects, the physiological response is a slow excretion of sodium, which can be impaired by hyperchloremia $[8,9]$. Because patients undergoing cystectomy are prone to have transient creatinine increase, electrolyte disturbance, and acidosis postoperatively, it would make sense to administer a more physiological intravenous solution to these patients perioperatively. In addition, it has been demonstrated that the influence of salt and water balance on gastrointestinal (GI) recovery after colonic surgery is clinically relevant: patients receiving less fluid and less sodium show faster recovery of GI function, resulting in a shorter hospitalization time [10]. This is of crucial importance in patients undergoing cystectomy because they are at risk for postoperative constipation or ileus.

The aim of this study is to evaluate, in a prospective, single-center, randomized, parallel-group, assessor-blinded study design, the physiology of electrolyte and water homeostasis in patients undergoing open radical cystectomy and urinary diversion, using two different fluid regimens. We hypothesize that an approach with a glucose/ potassium-based and reduced chloride crystalloid solution will result in faster return of GI function, reduced hypernatremia and hyperchloremic metabolic acidosis, less frequent hyperosmolality, and a better maintained fluid balance compared with a regimen using a balanced Ringer's crystalloid solution.

\section{Method and design} Ethics approval

The study was approved by the local ethics committee (Kantonale Ethikkommission Bern KEKBE; chairperson: Professor Dr N Tueller; registration number 151/13) on October 22, 2013. This study is conducted in accordance with the Declaration of Helsinki and was prospectively registered on November 21, 2013 at http://www.controlledtrials.com/isrctn with the trial identification number ISRCTN32976792.

\section{Objectives of the study}

The main objectives of this study are 1) to analyze if a positive salt and water balance is associated with a delayed return of GI function; 2) to compare arterial $\mathrm{pH}$, electrolyte $\left(\mathrm{Na}, \mathrm{K}, \mathrm{Cl}, \mathrm{Mg}, \mathrm{HPO}_{4}\right)$, glucose, and bicarbonate concentrations at 6 h, 24, 48, 72 and 96 hours postoperatively; 3 ) to measure sodium and water excretion over the same time period; 4) to study the effect of these crystalloid solutions on plasma osmolality, levels of brain natriuretic peptide (BNP), and plasma concentration of the hormones controlling water and sodium excretion (plasma renin, aldosterone, arginin-vasopressin); and 5) to measure the urine osmolality and concentration of $\mathrm{Na}, \mathrm{Cl}$ and neutrophil gelatinase-associated lipocalin (NGAL).

\section{Study endpoints}

The primary outcome variable is the postoperative return of GI function after open radical cystectomy, defined as the first defecation and expressed in days.

Secondary outcome variables are postoperative first flatus, positive fluid balance (body weight difference postoperatively versus preoperatively); incidence of kidney function disorders according to the RIFLE classification [11]; difference in $\mathrm{pH} 24$ hours postoperatively (metabolic acidosis defined as hyperchloremia, normal anion gap, low plasma bicarbonate); changes in plasma and urine osmolality during the duration of infusion; incidence of hypernatremia during the duration of infusion; incidence of hyperchloremia during the duration of infusion; incidence of hypokalemia or hyperkalemia during the duration of infusion; changes in plasma renin, aldosterone, arginin-vasopressin, and BNP levels; and changes in NGAL urine values.

\section{Study design}

This is a prospective, single-center, randomized, parallelgroup, interventional, assessor-blinded trial conducted at the Department of Urology of the Bern University Hospital, Switzerland. We expect to randomize the first patient in August 2014. 


\section{Blinding and randomization}

Assessment of the endpoint data will be performed in a blinded manner. The study nurse responsible for data acquisition will not be allowed to see the content of the crystalloid infusion. Laboratory, defecation, flatus, and body weight data will be assessed electronically in the nurse ward office using the CGM Phoenix Workstation 7-i-PDOS ${ }^{\odot}$; it will not be assessed in front of the patient.

Randomization will be carried out using a computergenerated list with 11 blocks of 4 patients per block. Allocation will be stored in sealed opaque and numbered envelopes. Patients will be included strictly in numerical order.

\section{Selection of the participants}

Consecutive patients with bladder cancer will be identified and recruited during the preoperative assessment of eligibility for an open radical cystectomy with urinary diversion. Participants fulfilling the inclusion criteria will be asked for their signed informed consent as required by the local ethics committee, and in accordance with the Declaration of Helsinki.

Inclusion criteria are age $\geq 18$ years old, type of surgery (open radical cystectomy with urinary diversion; that is, ileal conduit, orthotopic bladder substitute, catheterizable ileal pouch), American Society of Anesthesiologists (ASA) physical status II and III, and written informed consent.

Exclusion criteria are pregnancy, breast-feeding (which are exclusion criteria for this type of surgery itself), congestive heart failure (New York Heart Association (NYHA) classification $\geq 3$ ), severe hepatic disease (prothrombin ratio $<50 \%$ ), and significant renal dysfunction (estimated glomerular filtration rate $<45 \mathrm{ml} / \mathrm{min}$ ).

\section{Time course of the study and collection of the data Admission day}

After informed consent, patients undergoing open radical cystectomy with urinary diversion will be randomized, according to the concealed numbered envelopes. Preoperative blood samples will be taken (plasma samples: $\mathrm{Na}, \mathrm{K}, \mathrm{Cl}, \mathrm{Mg}, \mathrm{HPO}_{4}$, glucose, osmolality, renin, aldosterone, arginine-vasopressin, BNP; urine samples: NGAL, $\mathrm{Na}, \mathrm{Cl}$ and osmolality) and body weight documented. In addition, preoperative American Society of Anesthesiologists (ASA) physical status score, Charlson comorbidity score, and Glasgow prognostic score will be recorded.

Included patients will be requested to note the time of first flatus and to inform the ward nurses accordingly. During the first 5 postoperative days, patients will stay on the intermediate care unit; this will allow rapid assessment of the first flatus and defecation as patients will be continuously monitored and will have at least one visit by the nurse per hour. Assessment of the first flatus and defecation will be electronically documented (including time of event).

\section{$6 \mathrm{~h}$ postoperatively}

Blood samples will be taken (arterial blood gas analysis, plasma samples: $\mathrm{Na}, \mathrm{K}, \mathrm{Cl}, \mathrm{Mg}, \mathrm{HPO}_{4}$, glucose, osmolality, renin, aldosterone, arginine-vasopressin, BNP) as well as urine samples of NGAL, $\mathrm{Na}, \mathrm{Cl}$ and osmolality.

\section{Postoperative day 1}

Blood samples (arterial blood gas analysis, plasma samples: $\mathrm{Na}, \mathrm{K}, \mathrm{Cl}, \mathrm{Mg}, \mathrm{HPO}_{4}$, glucose, osmolality, renin, aldosterone, arginine-vasopressin, BNP) and urine samples (NGAL, $\mathrm{Na}, \mathrm{Cl}$ and osmolality) will be taken. Body weight, fluid balance (that is, difference between preoperative weight and weight on postoperative day 1), and assessment of flatus and defecation will be documented.

\section{Postoperative days 2 to 4}

On each of these days, blood samples (arterial blood gas analysis, plasma samples: $\mathrm{Na}, \mathrm{K}, \mathrm{Cl}, \mathrm{Mg}, \mathrm{HPO}_{4}$, glucose, osmolality, renin, aldosterone, arginine-vasopressin, BNP) and urine samples (NGAL, Na, $\mathrm{Cl}$ and osmolality) will be taken. Body weight and assessment of flatus and defecation will be documented.

\section{From postoperative day 5}

Body weight and assessment of flatus and defecation will be documented until return of the GI function (that is, defecation) has occurred.

Figure 1 shows the CONSORT diagram of the trial.

\section{Registration and record-keeping}

All data will be collected and recorded onto case report forms (CRFs) by a trained study nurse blinded to the randomization group, which will be recorded onto a secure electronic database in compliance with the new Swiss federal law on research on human subjects (Human Research Act, HFG/LRH) and related ordinances (KlinV/ OClin, HFV/ORH, and OV-HFG/Org LRH) and ICHGCP E6. Data assessment on CRF will be anonymous. In accordance with Swiss law, all original records (CRF and relevant correspondence) will be archived for 15 years in a secure locked room in the Department of Urology, Bern University Hospital, and then destroyed according to the hospital standards concerning destruction of confidential information. This will be carried out even if the trial is terminated prematurely.

\section{Data monitoring}

External, independent monitoring will be performed on site for quality control purposes by a study nurse trained in data monitoring and recording, who will not be involved in the study. This external monitoring will evaluate 


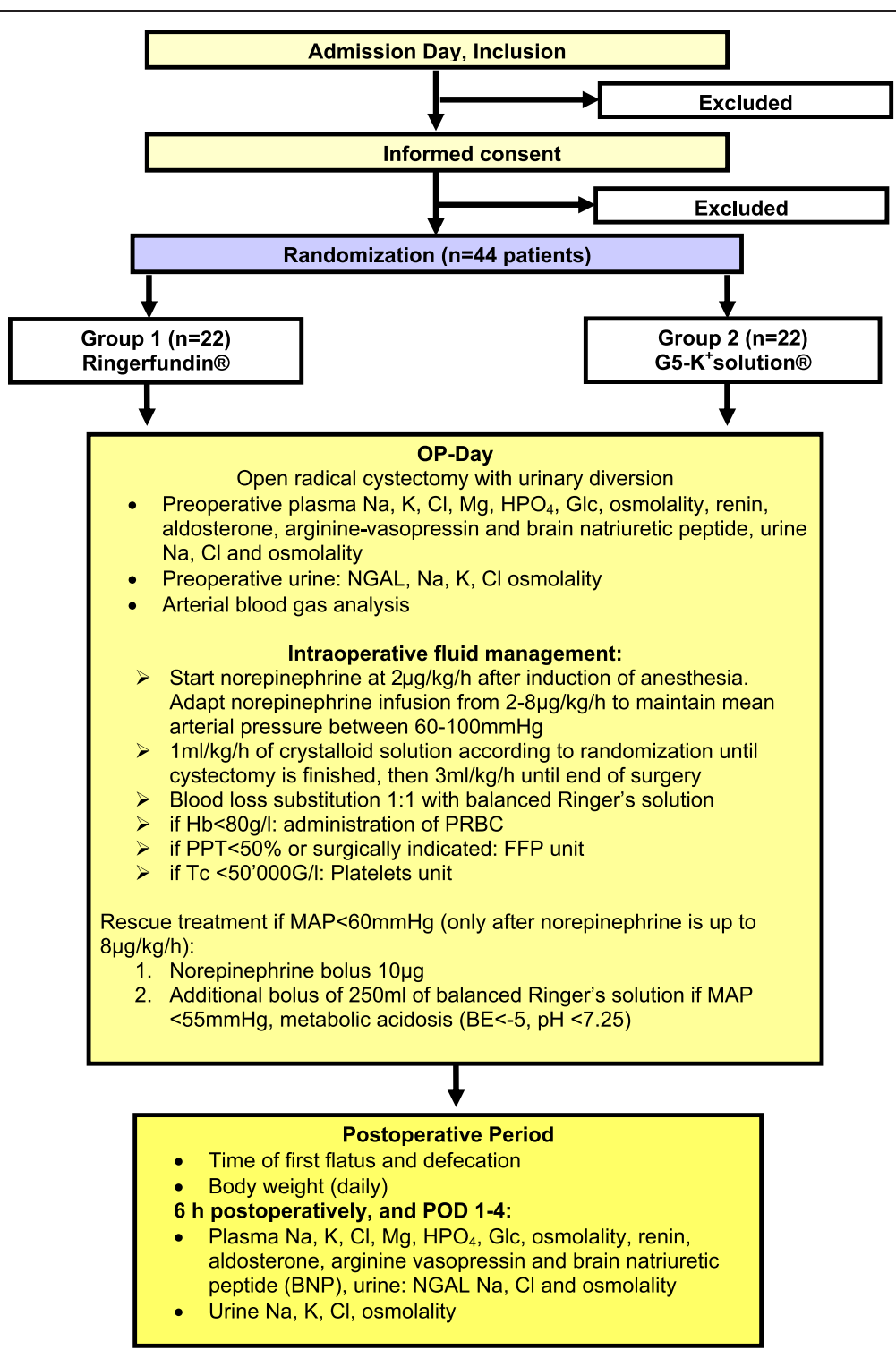

Figure 1 CONSORT diagram. BE, base excess; FFP, fresh frozen plasma; MAP, mean arterial pressure; PRBC, packed red blood cell.

the progress of the study, and verify the accuracy and completeness of the data recording (CRF). Two monitoring visits will be planned per year. Study data will be centrally monitored by a study nurse (Department of Urology, Bern University Hospital, Switzerland), who is not involved in the recruitment or randomization of patients, or assessment of the data. This study nurse will perform source data verification for all patients, including written informed consent, inclusion and exclusion criteria, and study outcomes.

\section{Study intervention}

An equal number of patients will be randomized to receive either Ringerfundin ${ }^{\circledR}$ solution or G5-K crystalloid solution as baseline infusion during the entire period that an intravenous administration of fluid is necessary (Table 1). The G5-K crystalloid solution is already used as a baseline infusion on our intensive care unit, and has been shown to be safe.

\section{Intraoperatively}

After induction of anesthesia, a concomitant norepinephrine infusion will be started at $2 \mu \mathrm{g} / \mathrm{kg} / \mathrm{h}$ until the end of surgery, and the randomized crystalloid solution (Ringerfundin ${ }^{\circledR}$; B Braun Medical AG, Sempach, Switzerland) for group 1 or the G5-K solution for group 2 (Bichsel, Interlaken, Switzerland) will be infused at a rate of $1 \mathrm{ml} / \mathrm{kg} / \mathrm{h}$ until the bladder has been removed, followed 


\begin{tabular}{|c|c|c|}
\hline Electrolyte & Ringerfundin ${ }^{\circledast}$ & G5-K solution \\
\hline Sodium & $145.0 \mathrm{mmol} / \mathrm{l}$ & $50.0 \mathrm{mmol} / \mathrm{l}$ \\
\hline Potassium & $4.0 \mathrm{mmol} / \mathrm{l}$ & $30.0 \mathrm{mmol} / \mathrm{l}$ \\
\hline Magnesium & $1.0 \mathrm{mmol} / \mathrm{l}$ & $2.0 \mathrm{mmol} / \mathrm{l}$ \\
\hline Calcium & $2.5 \mathrm{mmol} / \mathrm{l}$ & $0 \mathrm{mmol} / \mathrm{l}$ \\
\hline Chloride & $127.0 \mathrm{mmol} / \mathrm{l}$ & 0 mmol/l \\
\hline Acetate & $24.0 \mathrm{mmol} / \mathrm{l}$ & $0 \mathrm{mmol} / \mathrm{l}$ \\
\hline Maleate & $5.0 \mathrm{mmol} / \mathrm{l}$ & $0 \mathrm{mmol} / \mathrm{l}$ \\
\hline Lactate & $0 \mathrm{mmol} / \mathrm{l}$ & $18 \mathrm{mmol} / \mathrm{l}$ \\
\hline $\mathrm{HPO}_{4}$ & $0 \mathrm{mmol} / \mathrm{l}$ & $8.0 \mathrm{mmol} / \mathrm{l}$ \\
\hline Glucose & $0 \mathrm{mmol} / \mathrm{l}$ & $50 \mathrm{~g} / \mathrm{l}$ \\
\hline
\end{tabular}

by $3 \mathrm{ml} / \mathrm{kg} / \mathrm{h}$ until the end of surgery. If hypotension is observed (mean arterial pressure (MAP) $<60 \mathrm{mmHg}$ ), norepinephrine will be titrated to a maximum of $8 \mu \mathrm{g} / \mathrm{kg} / \mathrm{h}$ after an initial bolus of $10 \mu \mathrm{g}$. If hypotension persists, a bolus of $250 \mathrm{ml}$ of balanced Ringer's solution (Ringerfun$\operatorname{din}^{\circ}$ ) in both groups will be given.

In both groups, blood loss of $>500 \mathrm{ml}$ will be substituted with an equal amount of Ringerfundin ${ }^{\circ}$. Packed red blood cells (PRBC) will be transfused if hemoglobin values are less than $80 \mathrm{~g} / \mathrm{l}(<100 \mathrm{~g} / \mathrm{l}$ in patients with coronary artery disease). Additional boluses of Ringerfun$\operatorname{din}^{\circ}(250 \mathrm{ml})$ will be infused as a rescue medication if MAP persists at less than $60 \mathrm{mmHg}$ after the aforementioned correction, and in cases of severe intraoperative metabolic acidosis (base excess $<-5, \mathrm{pH}<7.25$ ) attributable to severe hypovolemia.

\section{Postoperatively}

Postoperative hydration will be identical in both groups, and will consist primarily of $1,500 \mathrm{ml}$ of the randomized crystalloid solution per 24 hours [10]. If MAP is less than $60 \mathrm{mmHg}$ after a bolus of $500 \mathrm{ml}$ of Ringerfundin ${ }^{\circ}$, norepinephrine will be infused up to a rate of $200 \mu \mathrm{g} / \mathrm{h}$ in both groups. PRBC units will be transfused according to ASA guidelines [12], and fresh frozen plasma units will be given if the prothrombin time is $>1.5$ times normal value.

In both groups, patients will be allowed to drink clear fluids immediately after surgery while on the intermediate care unit. An oral liquid diet will be started on POD 1 and patients will begin active mobilization. To enhance recovery of bowel function, patients will be treated strictly according to our postoperative enhanced recovery protocol (Table 2). Use of chewing gum will be encouraged [13], and the subcutaneous application of neostigmine $0.5 \mathrm{mg}$ will be started on POD
2 (Table 2). Body weight will be measured daily in the morning. Time of first flatus and first defecation will be recorded.

\section{Statistics}

Data will be analyzed by the research team in collaboration with a medically versed biostatistician. There will be no interim analysis.

Statistical analysis will be conducted on an intention-totreat basis. Data distribution will be tested by Q-Q plots. Normally distributed data will be presented as mean and standard deviation (SD), skewed data as median and range, and dichotome data as number and percentage. Comparing related and unrelated continuous samples, the paired and unpaired $t$-tests, respectively, will be used for normally distributed data and the Wilcoxon signed rank test and Mann-Whitney $U$-test, respectively for skewed data. Differences in proportions will be analyzed using the Fisher's exact test, and risk ratio with associated $95 \%$ confidence interval (CI). For the analysis of the primary endpoint, we will use the unpaired Student $t$-test with associated 95\% CI. In addition, a multiple logistic regression analysis will be carried out, including the randomization groups and all clinically relevant variables (age, ASA scores, Charlson comorbidity score, Glasgow prognostic score, postoperative fluid balance). A two-sided $P$ value of less than 0.05 will be considered significant.

\section{Sample analysis}

Based on retrospective data from our institution in the same surgical population (mean duration until first defecation 4.82 days, SD of 0.82 days), a power analysis was performed using duration of return of GI function as the primary variable. For a calculated sample size of 18 patients in each group (36 patients in total) the study is designed to have an $90 \%$ power $(\beta=0.10)$ for the Wilcoxon/ Mann-Whitney test to detect a difference of 1 day between the groups (Ringerfundin ${ }^{\circ}$ and $\mathrm{G} 5-\mathrm{K}$ solution) at a two-sided significance level of $5 \%(\alpha=0.05)$ assuming an SD of 1 day (G Power Version 3.1.5., Kiel and Duesseldorf, Germany). This decrease of 1 day is considered clinically relevant. Assuming a drop out of $20 \%, 22$ patients per group will be recruited.

\section{Assessment of safety}

The methods used to measure plasma and urine samples are those established at our hospital and used in everyday clinical practice. Both intravenous solutions will be provided by the pharmacy of the University Hospital Bern. Both solutions are routinely used at our institution (Department of Anesthesia and Department of Intensive Care Medicine). 
Table 2 Study relevant information about enhanced recovery program for cystectomy patients at the University Department of Urology, Bern (Cystectomy Enhanced Recovery Approach (CERA ${ }^{\odot}$ ))

\begin{tabular}{|c|c|}
\hline Time points & Interventions \\
\hline \multicolumn{2}{|l|}{ Preoperative } \\
\hline & No enteral bowel preparation \\
\hline & Two high enemas the evening before surgery \\
\hline & Normal nutrition till midnight before surgery \\
\hline & Clear drinks including carbohydrate till $2 \mathrm{~h}$ before surgery \\
\hline & Subcutaneous injection of low molecular heparin at 20:00 hours \\
\hline \multicolumn{2}{|l|}{ Intraoperative } \\
\hline & DVT prophylaxis with T.E.D. ${ }^{\text {TM }}$ hose or sequential compression devices \\
\hline & Perioperative antibiotics 30 min before surgical incision \\
\hline & Restrictive fluid regimen aiming at zero postoperative weight gain \\
\hline & Gastrostomy tube placed, removal of orogastric tube at end of procedure \\
\hline \multicolumn{2}{|l|}{ Postoperative } \\
\hline & $\begin{array}{l}\text { DVT prophylaxis with ambulation, T.E.D. }{ }^{\text {TM }} \text {, and subcutaneous low molecular heparin (weight adapted), started } 6 \text { hours } \\
\text { postoperatively }\end{array}$ \\
\hline & Chewing gum encouraged \\
\hline & Clear drinks allowed the same evening after surgery \\
\hline & $\begin{array}{l}\text { Gastrostomy tube initially left on drainage; closure of the gastrostomy tube will occur when patient is without nausea and vomiting } \\
\text { for }>24 \mathrm{~h}\end{array}$ \\
\hline & $\begin{array}{l}\text { Bedside mobilization as soon as possible, ideally the same evening after surgery, but if this is not possible, not later than the next } \\
\text { morning }\end{array}$ \\
\hline & Initial pain treatment with thoracic epidural analgesia, no opioids \\
\hline & Gl ulcer prophylaxis with esomeprazole for at least the first 2 POD \\
\hline & Antibiotics for 48 hours \\
\hline & Ambulation on POD 1 \\
\hline & Start oral fluids including energy drinks $\left(\right.$ Ensure $^{\oplus}$, Impact $\left.^{\oplus}\right)$ on POD 1 \\
\hline & Unrestricted clear drinks on POD 1 \\
\hline & Prokinetics: start with 0.5 mg neostigmin subcutaneously up to 4 times per day on POD 2 \\
\hline & Small snacks introduced on POD 2, not later than POD 3 \\
\hline & POD 3: encourage longer mobilization, walking distances, and spending time in a chair \\
\hline & Anti-emetics given only on request \\
\hline & Drains removed if draining $<50 \mathrm{ml} /$ day \\
\hline & Gastrostomy tube removed once the patient has passed stool \\
\hline & POD 5: thoracic epidural removed, oral analgesics (metamizole, paracetamol, hydroxycodon/naloxon $\left(\right.$ Targin $\left.^{\circledR}\right)$ ) \\
\hline
\end{tabular}

DVT, deep vein thrombosis; POD, postoperative day; T.E.D. ${ }^{\mathrm{T}}{ }^{\mathrm{M}}$, anti-embolism stockings by Covidien, Mansfield.

\section{Reporting of adverse events}

Follow-up will be guaranteed because every patient included in the trial will receive daily visits from the urologist in charge and will have blood samples taken. In addition, all patients will be continuously monitored during POD 1 to 5 , as they will be on the intermediate care unit.

The principal investigator (PYW) will monitor the patients for any adverse events (AEs), which are defined as any unfavorable and unintended sign (including abnormal laboratory results), symptom, or disease temporally associated with the use of the randomized infusions. This will be documented on the CRF and immediately communicated to the principal investigator.

Serious AEs (SAEs) are defined as any untoward medical occurrence at any dose that results in death, or is lifethreatening (i.e. patient was at risk of death at the time of the event), requires inpatient hospitalization or prolongation of existing hospitalization, or results in persistent or significant disability/incapacity. All treatment related to SAEs will be recorded and reported to the local ethics committee. An investigation will be conducted, and a 
report on the findings generated before the study can be resumed, in accordance with the Good Clinical Practice Guidelines of the Declaration of Helsinki.

\section{Duration and timeline}

The annual caseload of cystectomy is around 100 in our institution per year. We expect to recruit our participants within 18 months. The study protocol was designed and approval from the local ethics committee obtained in 2013, and participants will be recruited during 2014 and 2015.

\section{Discussion}

The present study will improve knowledge on the use of perioperative crystalloid solutions in patients with bladder cancer undergoing radical cystectomy with urinary diversion. This information should produce a basis on which to develop strategies that aim to prevent perioperative morbidity such as delayed return of the GI function and postoperative renal dysfunction.

The primary outcome of this study is the return of GI function, which is a cornerstone of the postoperative management of patients after major abdominal surgery. Delayed return of GI function is one of the major reasons for prolonged hospitalization time. The potential implications of our results are important. Accelerating the return of GI function will inevitably shorten hospitalization time and thus reduce the cost of hospital expenditure.

We expect by using the G5-K solution, which does not contain chloride anions, to achieve not only a near-zero water balance, as we already demonstrated in a previous $\mathrm{RCT}$, but also a near-zero salt balance after radical cystectomy with urinary diversion [1]. In contrast we expect the patients in the Ringerfundin ${ }^{\circ}$ group to be at higher risk of having a positive fluid and salt balance. This nonaccumulation of salts and water in the G5-K solution group is expected to result in an earlier return of the GI function. Lobo et al. already showed that a positive salt and water balance after colonic resection delayed the return of GI function and resulted in longer hospitalization time. In addition, perioperative chloride loading is known to impair urinary output, and to induce metabolic acidosis and even hyperkalemia in patients with preoperative renal dysfunction.

\section{Conclusion}

In conclusion, this trial is an investigator-initiated, pragmatic, randomized clinical trial to test the hypothesis that administration of glucose-rich balanced crystalloid solution containing potassium can accelerate the return of GI function. In addition, this study will also determine the effect of this solution on renal function after radical cystectomy with urinary diversion.

\section{Trial status}

The trial is ongoing.

\section{Abbreviations}

ASA: American Society of Anesthesiologists; BNP: Brain natriuretic peptide: Cl: confidence interval; CRF: case report form; Gl: gastrointestinal; MAP: Mean arterial pressure; NGAL: Neutrophil gelatinase-associated lipocalin; PRBC: Packed red blood cells; POD: postoperative day; RCT: Randomized clinical trial; RIFLE: risk, injury, failure, loss, end-stage renal disease; SD: standard deviation.

\section{Competing interests}

The authors declare that to have no competing interests.

\section{Authors' contributions}

PYW/LML/BK drafted the manuscript together with FCB. Study designed by all authors. Logistic organization will be supported by PYW and FCB. All authors read and approved the final manuscript.

\section{Acknowledgements}

We thank Professor J Takala, MD, Chairman of the Department of Intensive Care Medicine, University Hospital Bern, for his important intellectual support. This study is supported by internal departmental funding of the Department of Anesthesiology and Pain Medicine and Department of Urology, University Hospital Bern, Switzerland.

\section{Author details}

${ }^{1}$ Department of Anesthesiology and Pain Medicine, Inselspital, Bern University Hospital, CH-3010 Berne, Switzerland. Department of Urology, Inselspital, Bern University Hospital, Bern, Switzerland.

Received: 1 May 2014 Accepted: 23 June 2014

Published: 8 July 2014

\section{References}

1. Wuethrich PY, Burkhard FC, Thalmann GN, Stueber F, Studer UE: Restrictive deferred hydration combined with preemptive norepinephrine infusion during radical cystectomy reduces postoperative complications and hospitalization time: a randomized clinical trial. Anesthesiology 2014, 120(2):365-377.

2. Brandstrup B, Tonnesen H, Beier-Holgersen R, Hjortso E, Ording H, Lindorff-Larsen K, Rasmussen MS, Lanng C, Wallin L: Effects of intravenous fluid restriction on postoperative complications: comparison of two perioperative fluid regimens. Ann Surg 2003, 238(5):641-648.

3. Prowle JR, Chua HR, Bagshaw SM, Bellomo R: Clinical review: volume of fluid resuscitation and the incidence of acute kidney injury - a systematic review. Crit Care 2012, 16(4):230.

4. Yunos NM, Bellomo R, Hegarty C, Story D, Ho L, Bailey M: Association between a chloride-liberal vs chloride-restrictive intravenous fluid administration strategy and kidney injury in critically ill adults. JAMA 2012, 308(15):1566-1572.

5. Yunos NM, Bellomo R, Story D, Kellum J: Bench-to-bedside review: chloride in critical illness. Crit Care 2010, 14(4):226.

6. Thakar CV: Perioperative acute kidney injury. Adv Chronic Kidney Dis 2013, 20(1):67-75

7. Borthwick E, Ferguson A: Perioperative acute kidney injury: risk factors, recognition, management, and outcomes. BMJ 2010, 341:c3365.

8. Williams EL, Hildebrand KL, McCormick SA, Bedel MJ: The effect of intravenous lactated Ringer's solution versus $0.9 \%$ sodium chloride solution on serum osmolality in human volunteers. Anesth Analg 1999, 88(5):999-1003.

9. Lobo DN, Stanga Z, Aloysius MM, Wicks C, Nunes QM, Ingram KL, Risch L, Allison SP: Effect of volume loading with 1 liter intravenous infusions of $0.9 \%$ saline, $4 \%$ succinylated gelatine (Gelofusine) and $6 \%$ hydroxyethyl starch (Voluven) on blood volume and endocrine responses: a randomized, three-way crossover study in healthy volunteers. Crit Care Med 2010, 38(2):464-470.

10. Lobo DN, Bostock KA, Neal KR, Perkins AC, Rowlands BJ, Allison SP Effect of salt and water balance on recovery of gastrointestinal function after elective colonic resection: a randomised controlled trial. Lancet 2002, 359(9320):1812-1818. 
11. Hoste EA, Clermont G, Kersten A, Venkataraman R, Angus DC, De Bacquer D, Kellum JA: RIFLE criteria for acute kidney injury are associated with hospital mortality in critically ill patients: a cohort analysis. Crit Care 2006, 10(3):R73.

12. Klein $H G$, Spahn DR, Carson JL: Red blood cell transfusion in clinical practice. Lancet 2007, 370(9585):415-426.

13. Kouba EJ, Wallen EM, Pruthi RS: Gum chewing stimulates bowel motility in patients undergoing radical cystectomy with urinary diversion. Urology 2007, 70(6):1053-1056.

doi:10.1186/1745-6215-15-276

Cite this article as: Löffel et al:: Perioperative use of crystalloids in patients undergoing open radical cystectomy: balanced Ringer's maleate versus a glucose $5 \% /$ potassium-based balanced solution: study protocol for a randomized controlled trial. Trials 2014 15:276.

\section{Submit your next manuscript to BioMed Central and take full advantage of:}

- Convenient online submission

- Thorough peer review

- No space constraints or color figure charges

- Immediate publication on acceptance

- Inclusion in PubMed, CAS, Scopus and Google Scholar

- Research which is freely available for redistribution 